\title{
La renaissance des représentations sur la femme en Afrique contemporaine. De la posture victimaire à l'héroïne dans les productions discursives et artistiques en RD Congo
}

\author{
Jean-Claude MAPENDANO BYAMUNGU ${ }^{1}$ \\ Gratien LUKOGHO VAGHENI ${ }^{2}$
}

\begin{abstract}
Africa is fundamentally a patriarchal society. And the social discourses circulating in this continent underline representations with very demeaning negative stereotypes towards the women. This is, indeed, a behavioral and linguistic credo that has long frozen women in a victimwood posture. Today, the situation is shifting towards a certain renewal of this a priori sexist collective imagination. The challenge is to build a heroine posture that now represents women as the pillar of Africa's socio-economic development in the dynamics of contemporary sustainable development goals. Thus, this study analyzes through a diversified corpus which takes into account both paremic orality, literary production and ordinary discourse, the socio-discursive mechanisms of this discourse of societal issues in a rapidly changing $R D$ Congo.
\end{abstract}

Keywords: discourse analysis ; heroine ; victim posture ; renaissance ; representations DOI: $10.24818 / \mathrm{DLG} / 2021 / 38 / 12$

\section{Introduction}

$\mathrm{D}$ epuis près de vingt siècles déjà, tous les discours de la femme et sur la femme en Afrique contemporaine l'ont toujours figée dans une posture victimaire dans ses rapports avec son partenaire masculin. Dans le sillage de la politique, les femmes constituent encore, à quelques exceptions près, une force folklorique de mobilisation électorale pour les hommes. À l'ores de ce $21^{\text {ème }}$ siècle, cette image d'éternelle victime jadis stéréotypée dans son rôle protocolaire est en train d'évoluer vers celle d'une héroïne c'est-à-dire celle de solution ultime aux problèmes sociétaux

\footnotetext{
${ }^{1}$ Jean-Claude Mapendano Byamungu, ISP de Kichanga/RD Congo, jcmapendanos26@gmail.com

${ }^{2}$ Gratien Lukogho Vagheni, ISP de Kalehe/RD Congo, vlukogho@gmail.com
} 
en général et de sa famille nucléaire en particulier. En RD Congo, l'image de plus en plus mise en scène dans les discours sociaux que sous-tendent ces représentations aux déterminismes socioculturels et politiques, est désormais celle d'une Jeanne d'Arc afro-congolaise, capable de tout réussir là où les hommes sont en train de lamentablement échouer. Elle s'actualise en slogans de propagande sociétale, en expressions figées ou sous le prisme de l'art pictural à connotation méliorative sur la femme. C'est entre autres les énoncés tels que "Avec la femme, la communauté gagne», "Avec la femme, il est encore possible », "La force de l'Afrique », "Elles peuvent faire mieux", "Rien sans les femmes », etc.

Cette étude fonde son objet sur une analyse des mécanismes sociodiscursifs qui cristallisent les représentations de la femme, ancrées dans un renouvellement des stéréotypes négatifs et réducteurs (posture victimaire) pour la fabrique d'une posture plutôt d'héroïne dans l'imaginaire collectif. De ce point de vue, elle entend répondre aux interrogations ci-dessous : Comment la posture victimaire de la femme estelle mise en discours dans le corpus? Quelle est la posture de l'hérö̈ne en construction et sur laquelle repose l'hypothèse d'une renaissance des représentations sur la femme et pour quels enjeux sociétaux? Ainsi exploite-elle un ensemble des données empiriques issus des corpus divers, entre autres, l'orature parémique, la production littéraire, l'art pictural, bref un ensemble de discours sociaux en circulation sur divers types de médias.

Dès lors, elle adopte le parti pris de s'inscrire dans le champ interdisciplinaire de l'Analyse du Discours (AD) à la française. Depuis son émergence, l'AD a été diversement définie selon les auteurs, entre autres Brown et Yule (1983), Van Dijk (1985. En ce qui nous concerne, nous avons décidé de fonder notre propos sur la perspective de l'AD à la française, telle que l'objet et la démarche ont été circonscrits par Maingueneau (2009: 33) en ces termes :

«Pour notre part (Maingueneau, 1995), nous jugeons préférable de spécifier l'analyse du discours comme la discipline qui, au lieu de procéder à une analyse linguistique du texte en lui-même ou à une analyse sociologique ou psychologique de son "contexte", vise à rapporter les textes, à travers leurs dispositifs d'énonciation, aux lieux sociaux qui les rendent possibles et qu'ils rendent possibles. En conséquence, l'analyse du discours accorde un rôle clé aux genres de discours, qui ne sont pas considérés comme des types de textes, dans une perspective taxinomique, mais comme des dispositifs de communication, de nature à la fois sociale et linguistique. » 
Cette définition met en évidence la nécessité d'appréhender les différents genres de discours dans leur articulation aux contextes c'est-àdire aux lieux sociaux qui les génèrent. Ceux-ci sont à leur tour envisagés comme «les paramètres extérieurs situationnels » (Maingueneau, 2009 : 33). C'est pourquoi, selon Maingueneau (2009 : 33) toujours, la prise en compte $\mathrm{du}$ «contexte" d'émergence des discours est un élément central pour l'interprétation:

"Le contexte joue un rôle essentiel dans la production comme dans l'interprétation des énoncés: hors contexte, un énoncé n'a qu'un sens potentiel. Le contexte de quelque chose, de manière informelle, c'est ce qui l'environne. S'il s'agit d'une unité linguistique (phonème, mot, phrase, texte), cet environnement est à la fois linguistique (environnement verbal ou contexte) et non linguistique (environnement physique immédiat, mais aussi social ou culturel). On a tendance aujourd'hui à privilégier le terme «contexte » quand il s'agit de désigner l'environnement non verbal. »

Bref, le discours est toujours indissociable de son contexte. De façon plus pratique, il faudra cerner selon quelles modalités les discours renvoient au monde qui les génère à partir d'un processus inférentiel de dévoilement de leurs enjeux. Cependant, il sera question d'une analyse de contenu, en tant que méthodologie qualitative utilisée en sciences sociales et humaines (sociologie, linguistique, communication, psychologie) et qui tente de saisir la dimension contextuelle des textes, discours ou énoncés analysés afin de ressortir leurs déterminants sociaux dans une sémiosphère bien déterminée. Elle a aussi été diversement définie, notamment par Bardin (1977), Ghiglione et Beauvois (1980). Néanmoins, nous ne retiendrons que cette définition selon laquelle l'analyse de contenu correspond à « un terme générique désignant l'ensemble des méthodes d'analyse de documents, le plus souvent textuel, permettant d'expliciter le ou les sens qui sont contenues et/ou le ou les manières dont ils parviennent à faire effet de sens » (Muchielli (1996:36).

Aussi optons-nous, en ce qui concerne l'analyse de contenu, non seulement pour une analyse descriptive afin de déterminer avec plus ou moins de détails les éléments ainsi que les catégories du corpus mais aussi pour l'analyse verticale afin de dévoiler ses conditions de production. Partir du contenu manifeste au contenu latent, nous permet déterminer in fine les intentions explicites et implicites de la construction discursive d'une certaine renaissance vers un imaginaire collectif féministe en RD Congo. 
Dans son cheminement, la démarche sera menée en deux points. Le premier volet porte sur la configuration discursive de la posture victimaire de la femme alors que le second s'intéresse aux formes et enjeux de la posture de l'héroïne comme acte de renaissance des représentations sur la femme.

\section{La posture victimaire de la femme dans les discours sociaux en RD Congo}

L'Afrique est un continent à tradition patriarcale. Hérité de cette tradition aux coutumes rétrogrades, l'imaginaire collectif sur la femme peut se définir comme "un credo comportemental où prédomine la relation de méfiance, d'agression et la logique de l'extermination » (Makomo, 2014 : 94). Il s'agit tout d'abord d'un credo de comportement langagier, porté par un discours aux clichés réducteurs et de négativité sur la femme. Et il se traduit par la suite en pratiques discriminatoires et féminicides dont le viol accompagné parfois de la destruction des organes génitaux de la femme en reste l'une des formes les plus poussées jusqu'au paroxysme. Ce sont des discours médiés par divers types de corpus ainsi que des pratiques sociales qui ont incrusté en la femme un redoutable complexe d'infériorité au point de la figer dans une posture de victime expiatoire, jusqu'à la résignation.

\subsection{L'oralité parémique comme vecteur de la posture victimaire de la femme}

En Afrique en général et en RD Congo en particulier, le poids de la tradition a été longtemps utilisé comme argument pour justifier le credo comportemental sexiste et la discrimination des hommes vis-à-vis des femmes. Et le patrimoine parémique en est le principal vecteur. En effet, les proverbes constituent un riche patrimoine culturel de toute société à tradition orale. Cette forme de littérature africaine jouit du prestige de vecteur de la sagesse d'un peuple. De ce fait, elle constitue une sorte de cadre législatif ayant institué ce credo qui inféode la femme au pouvoir phallocratique masculin, l'assujettissant ainsi, depuis des siècles, au joug du système patriarcal.

À titre d'exemple, chez les Congolais de culture lingalaphone, l'image de la femme est celle d'une sorcière vis-à-vis de laquelle on doit garder une certaine distance sociale. Elle s'actualise essentiellement dans l'adage populaire «Kolia na mwasi kolia na ndoki» (Manger avec une femme 
$c^{\prime}$ est manger avec une sorcière). Pour ceux de culture swahiliphone de l'Est, le discours parémique consiste à prôner aussi la méfiance à l'égard de la femme perçue comme une menace. Les exemples que nous avons retenus sont notamment: "Mwanamuke hasemake katikati ya wanaume» (La femme ne parle pas parmi les hommes), "Banamuke haba kuwake ba ndugu yetu » (Les femmes ne sont pas nos sœurs).

Chez les Bashi du Sud-Kivu en outre, il y a lieu de citer l'équivalent du proverbe français «Toute femme a quatorze ans ». Il s'agit du proverbe «Omukazi arhakula » qui signifie littéralement « La femme ne grandit jamais » et glosable en "Toute femme est idiote » ou encore "Toute femme est bête », c'est-à-dire sans sens d'esprit critique et de discernement responsable. Mais il faut citer aussi le cas de «Omukazi akahimba anacijire nahano » (Si la femme vit dans l'aisance, elle finit par se substituer à son mari). Par ailleurs, les exemples empruntés au patrimoine culturel de la parémie des Bahavu d'Idjwi et de Kalehe (Sud-Kivu) décrivent une image de femme « mauvaise », « voleuse », " pire que le diable », « sans droit à l'éducation » et qui «ne doit pas hériter, gouverner ou occuper un poste de responsabilité », une simple "machine de procréation». Il en va de quelques énoncés proverbiaux ci-dessous :

- Abakazi boshi bishambo [Toutes les femmes sont des voleuses].

- Nta mikazi mwinja [Toutes les femmes sont mauvaises].

- Omukazi arha yima [La femme ne gouverne/n’hérite pas].

- Nta kinja chirhenga omu mukazi arhali omwana [De la femme, on ne peut rien attendre de bien sauf l'enfant].

- Amasomo g'omukazi gahwera omu mafiga [Les études de la femme finissent à la cuisine].

- Omukazi y'emwenge oku muzimu [La femme est plus rusée, pire que le diable]

Dans cette même logique de qualification péjorative du sexe féminin, nous pouvons mentionner pour le compte du patrimoine culturel nande (Banande/Nord-Kivu) des énoncés comme « Omukali ni waghu umuli oko njingo » (La femme est tienne lorsque vous êtes au lit), «Wamaha omukali uwalumako » (Si tu donnes quelque chose à la femme, il faut la grignoter un peu, comme pour dire qu'il ne faut jamais tout donner à une femme, il faut une certaine réserve vis-à-vis d'elle). Dans le langage ordinaire des Congolais, il existe aussi l'expression figée "un royaume de femmes", actualisée pour désigner un lieu où règne un désordre indescriptible, un «capharnaüm» ou alors un groupe sans leadership. Il ne s'agit que de 
quelques cas choisis de manière tout à fait aléatoire, mais le phénomène est généralisable à l'orature parémique de toutes les communautés ethnolinguistiques de la RD Congo.

Dans l'ensemble, il s'agit des proverbes très dévalorisants allant jusqu'au mépris. Actualisés dans le langage ordinaire quotidien, ces proverbes véhiculent des "stéréotypes» négatifs, le plus souvent considérés comme des vérités tranchantes car ils fonctionnent réellement comme des "prêts à porter de l'esprit " (Amossy, 1991 : 9). Du fait que le "stéréotype " est indissociable de la «représentation sociale» au sens psychologique du terme, la notion de "stéréotypage » est à évoquer ici, en tant qu'elle est «l'opération qui consiste à penser le réel à travers une représentation culturelle préexistante, un schème collectif figé » (Amossy, $2010: 45)$.

Désormais, tous les jugements en circulation sur la femme et le féminin dans les sociétés des cultures congolaises ont pour fondement les déterminismes socioculturels véhiculés par ces proverbes. Et ces jugements péremptoires répondent bien à la description $\mathrm{du}$ processus de « stéréotypage » présentée par Amossy (2010: 45) de manière ci-après :

"Il s'agit d'un imaginaire d'époque qui comprend un stock d'images toutes faites, de représentations collectives partagées par une communauté, ou tout du moins connues d'elle. La notion de représentation telle que l'utilise Grize est proche de ce que les psychologues sociaux appellent "représentation sociale», ou "stéréotype ». Le stéréotype peut être défini comme une image collective figée, qu'on peut décrire en attribuant un ensemble de prédicats à un thème (...). »

Notre point de vue pose cependant le postulat selon lequel l'émergence des préjugés réducteurs et dévalorisants sur la femme en Afrique relève sans aucun doute d'une construction sociale qui, au cours de l'histoire de l'humanité, a beaucoup plus opéré une lecture sexiste, subjective, sélective, biaisée et décontextualisée, en ne médiatisant qu'une facette du fonds culturel afro-congolais qu'est le patrimoine parémique. En effet, l'exploration objective du patrimoine proverbial dans le vaste champ de l'oralité de certains peuples (ethnies/tribus) de la RD Congo dévoile une autre face de ce patrimoine, longtemps occultée. Elle véhicule des représentations culturelles positivant la femme et le féminin. Nous pouvons citer, à titre purement indicatif qui ne prétend à aucune représentativité, des exemples de proverbes tels que : «Omukazi y'eyumbaka 
omulala» (La femme est le pilier bâtisseur de la communauté), "Omukazi y'ewabo muntu » (Il n'y a pas de meilleure partenaire que la femme) chez les mêmes Bahavu du Sud-Kivu auxquels nous avons déjà fait allusion.

À l'origine d'un imaginaire aux préjugés négatifs et dévalorisant sur la femme en Afrique, se trouve donc la lecture partielle et sexiste de l'orature du continent, en l'occurrence du discours parémique. Il s'agit d'une lecture masochiste entretenue par un courant traditionnel phallocratique, qui a perpétué le système patriarcal de générations en générations, avec comme conséquence idéologique l'institutionnalisation de l'infériorité sociale de la femme par rapport à son partenaire masculin. C'est un comportement langagier qui travaille à pérenniser les rapports de domination de la femme par l'homme. En somme, le discours proverbial d'Afrique le plus médiatisé sur la femme est un discours fondamentalement clivé et enclin aux stéréotypes de genre.

Mais il existe aussi chez eux une certaine idée selon laquelle «Les orphelins de mère survivent bien alors que ceux de l'homme ne survivent pas». C'est ce que nous lisons dans le proverbe havu «Enfubi z'omulume zirha kula» (Les orphelins de père ne survivent pas). Et en ce qui concerne la sagesse $\mathrm{du}$ peuple nande (Banande) du Nord-Kivu, nous avons également pris en compte les deux proverbes ci-dessous :

- Omukali ni mutima we nyumba, no mumlume ni kaliki ka erimbala omo mbidi [La femme est le cœur du foyer et l'homme est une corde à lier les reins].

- Avakali simuli mtokoro [Parmi les femmes, il n'y a pas de «mutokoro » (mutokoro : une partie de la cime de canne à sucre moins sucrée)].

Il s'agit des proverbes qui mettent en avant aussi bien la valeur de la femme en général que l'incontournable complémentarité partenariale entre l'homme et la femme. Pour qu'elle soit mise en valeur, il nécessite une relecture objective du discours proverbial africain afin de mieux la circonscrire, en l'articulant par exemple, aux orientations épistémologiques du féminisme africain, tel que l'envisage Chimamanda Ngozi Adichie, entre autres. En effet, leur contenus explicites et implicites recoupent parfois les enjeux et préoccupations de l'idéal féministe en Afrique subsaharienne. Tout ce comportement langagier de minoration de la femme décrit dans ces exemples se traduit parfois en actes de violences, physiques ou non, basées sur le genre, entre autres le viol utilisé comme arme de guerre dans le contexte des conflits armés en RD Congo. C'est un 
rituel des crimes aux pratiques féminicides que relaie un certain discours social à dominante idéologique antiféministe ou alors "féminosceptique » ou « gynosceptique » pour tenter quelques néologismes.

\subsection{La production littéraire comme discours contestataire de la posture victimaire de la femme}

Le domaine des discours est un champ qui se diversifie au fil de temps, par rapport aux nouveaux enjeux sociaux, spécifiques à une époque donnée. Le contexte des conflits armés inhérent à la guerre « internationale » qui déchire le pays depuis 1996 est au cœur d'une partie des discours dont nous nous servons ici pour la constitution du corpus. Il a ainsi élargi les types de violences dont la femme était le plus souvent victime dans le silence absolu. Des violences conjugales, on est passé à des cas systématiques de mutilations sexuelles, au viol collectif devenu une arme de guerre et de destruction massive. Le tout constitue autant de pratiques féminicides qui ne font que figer la femme dans sa posture victimaire et la contraindre en même temps à la résignation, voire à la négation de soi.

Dans le champ de la littérature négro-africaine francophone de la région des Grands Lacs dont fait partie la RD Congo (Burundi, RD Congo, Rwanda), les écrivains ont alors mis sur le marché du livre une production riche et diversifiée, aux titres évocateurs. Ceux-ci traduisent en mots, explicitement ou implicitement, le relent de sang symptomatique d'une mémoire discursive traumatique de violences à l'égard de la femme. Et le viol individuel ou collectif en reste le crime absurde, absolu. Les viols et violences de tous ordres sont alors les caractéristiques du destin fatal de la femme. C'est sous ce prisme déformant de «carrefour des ténèbres » que l'Est de la RD Congo vend son image au sein de l'opinion internationale. L'horrible tableau que nous fait Makomo Makita J.C. (2014 : 25) précise que « l'Est de la R.D. Congo est dénommé la capitale mondiale des viols et violences contre la femme et les bourreaux viennent de tous les pays des Grands Lacs ». C'est dans ce sens que, sensible à cette situation désespérée, la Présidente de la confédération suisse, Micheline Caliny-Rey, cité par Juvet (2011), puis reprise par Makomo Makita (2014 : 25), déclare en 2011 :

"J'ai vu des femmes magnifiques, des femmes victimes de ce conflit armé, des femmes qui subissent, quotidiennement, des horreurs abominables, qu'elles osent évoquer. Ces violences sont innombrables, ignobles. Le terme de violence est-il approprié ou est-ce plus que cela? Ces atteintes 
symptomatiques à ces femmes et enfants ne constituent pas une violation majeure de leurs droits humains mais font partie d'une stratégie de destruction d'une communauté toute entière et de son devenir. "

Dans un univers d'oppression à l'égard de la femme et de volonté manifeste de destruction du "genre féminin », les termes foisonnent pour décrire ou nommer ces horreurs abominables où l'homme a perdu tout sens d'humanisme. Nous ne nous limiterons ici qu'à illustrer notre propos par quelques titres caractéristiques de ce contexte. Le titre comme élément central du "péritexte " (Maingueneau, 2009: 78) nous semble essentiel à ce point, car il est le premier lieu à partir duquel le lecteur entre en contact avec le texte. Le romancier Bolya Baenga nous donne une belle métaphore pour nommer la pratique ignoble de viol contre la femme à travers le titre de son roman La profanation des vagins (2006). Faustin Muliri Miruho nous a enrichi de deux recueils de poèmes à savoir Attestation de perte de vagins (2009) et Solde à la vie de Femme (2011). D'autres énoncés titriques de notre corpus présentent l'image de la femme-victime en train de souffrir le martyre que lui impose le destin. Elle mène en cela une vie d'enfer qui a inspiré à Kabeya Mukamba le titre de son œuvre dramatique Anifa ou Même en enfer (2001).

Face à un drame qui dure depuis des années, l'écrivain nous présente une description cathartique qui demeure néanmoins une invitation à l'action ou à la prise de conscience. Il en exprime un sentiment de révolte, un cri de détresse, une sonnette d'alarme devant la situation d'une RD Congo où l'on viole et tue dans l'indifférence générale. Le viol des milliers de femmes dans la protubérance des conflits armés en RD Congo est alors à la base de l'infirmité physique et psychologique dont souffre désormais la femme dans la déchéance de sa féminité et de son humanité. C'est ce qui constitue la toile de fond du recueil de poèmes Infi (r)niment Femme (2009) de Bestine Kazadi Ditabala. Mais aussi le problème de la violence ménagère contre la femme constitue l'essentiel de la trame événementielle du roman Le destin détourné ou le journal d'une africaine molestée (1998) de Malu Bungi Mvenyi. Bref, le tableau est très atroce.

Le discours est donc celui de l'appel à la prise de conscience féministe, à l'engagement contre un système patriarcal prédateur. C'est dans cette optique qu'il faut lire la pièce de théâtre Ton combat, femme noire (1986) de Katende Kashi M'bika ou alors l'essai Les Fondements bibliques de la parité Homme-Femme (2014) de la journaliste Astrid Mujinga. L'écriture 
devient ainsi témoignage dans la détermination de l'écrivain à lever le voile du silence sur l'impunité des violences perpétrées contre des «victimes silencieuses» dont l'homme reste le triste bourreau en République Démocratique du Congo. La stratégie discursive adoptée ici est celle de la « qualification péjorative » comme fondement d'un discours contestataire.

\subsection{La posture victimaire de la femme dans la représentation chorégraphique}

La posture victimaire de la femme dans la représentation chorégraphique concerne essentiellement le métier des danseuses. En effet, la chorégraphie de la musique profane congolaise est un autre lieu par excellence de la mise en scène de la vulnérabilité de la femme au métier de danseuse. Elle concerne bien évidemment le type de tenue vestimentaire qui est le plus souvent imposé aux danseuses par les chefs d'orchestre. C'est un «dress code» d'une extravagance déconcertante, consistant à mettre en relief certaines parties intimes de la femme. La tenue tout comme les gestes chorégraphiques dans des clips sont d'une obscénité inouie au point que certains de ces clips font parfois l'objet de censure de la part de l'autorité de régulation. Les mélomanes y découvrent des jeunes filles à moitié nues et simulant quelques fois les rapports sexuels sur scène. C'est donc une pratique qui ne respecte aucun code éthique du respect du corps d'autrui, notamment du corps féminin.

L'hypothèse d'une posture victimaire repose sur le fait que le corps de la femme, si pas la femme elle-même, se trouve exploité comme un objet de marketing pour l'album. En plus, rien de tout cela ne relève du choix des danseuses. D'ailleurs leur métier est toujours associé à l'image des prostituées. Mais aussi beaucoup d'internautes ne se réservent pas d'assimiler la pratique au viol toujours décrié à l'Est du pays. Les deux images ci-dessous, publiées par des internautes congolais sur leurs pages Facebook, représentent les danseuses de l'artiste musicien Robinho Mundibu de Kinshasa en pleine séance de répétition : 


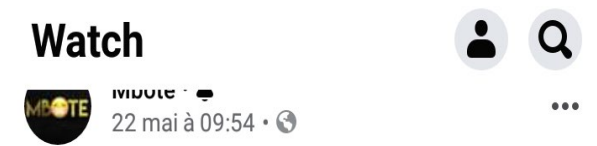

Et tes jaloux. Ils sont où ça ? $\circlearrowright \circlearrowright \circlearrowright$ Robinio Mundibu

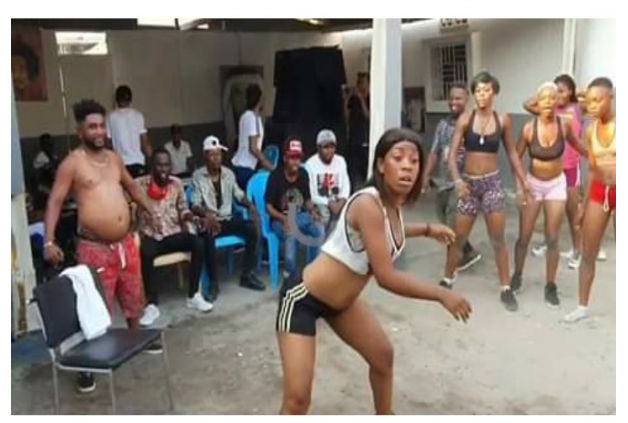

(1) 38071

845 commentaires $\cdot 3480$ partages

ㄱ' J'aime

$\checkmark$ Commenter

$\Rightarrow$ Partager
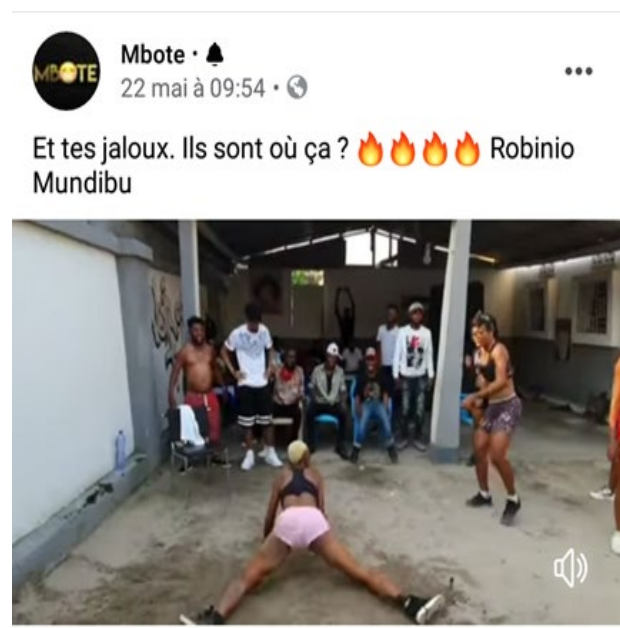

(1) 38071845 commentaires $\cdot 3480$ partages

0 J'aime $\square$ commenter $\Rightarrow$ Partager

Figure 2. Musicienne de Robinho

Les deux autres métadonnées ci-après, prises à partir des comptes de groupes du même contexte du réseau social Facebook, concernent plutôt les danseuses du célèbre artiste Jean-Noëlle Ngiama Makanda, dit Werrason.

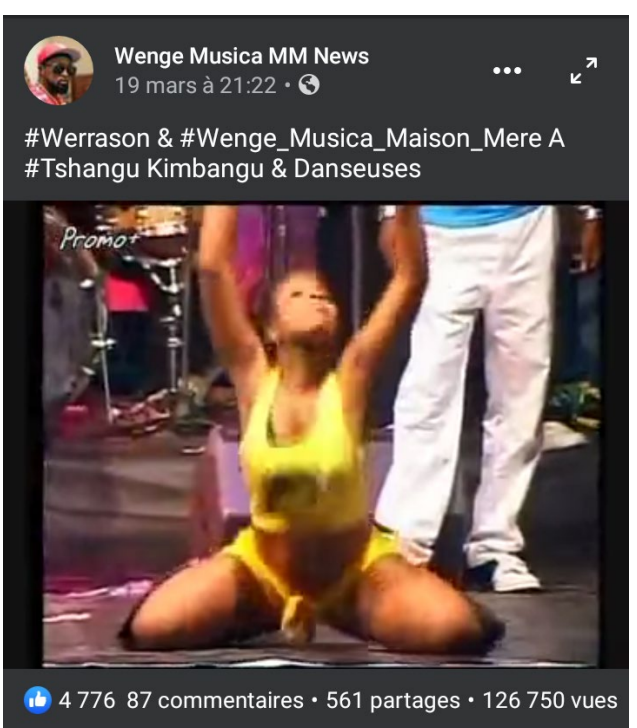

Figure 3. Danseuse de Werrason

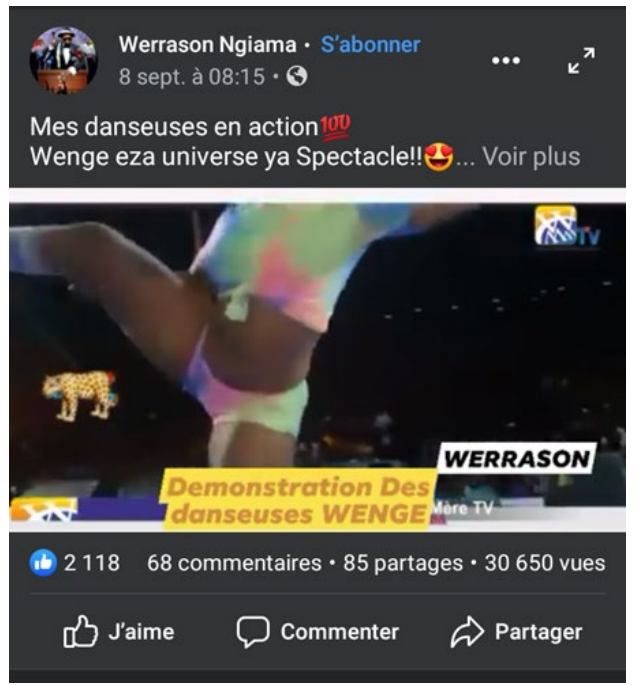

Figure 4. Danseuse de Werrason 
La situation des musiciennes décrites dans les images ci-dessus n'illustre pas un tableau valorisant de la femme. Il s'agit plutôt des scènes d'exposition publique de l'obscénité. C'est ce qui permet à une certaine opinion d'associer les différents chefs d'orchestres de Kinshasa aux violeurs de l'Est. Le discours socioculturel et politique qui accompagne ce genre de pratiques dégradantes à l'égard de la femme consiste, en quelque sorte, en la réification de celle-ci en ne lui assignant que le statut de comparse c'est-à-dire de figurant au sein de la méga-entreprise musicale dont elle sert alors d'élément du décor markétique.

\subsection{La posture victimaire de la femme au prisme de l'art pictural à Goma}

L'art pictural comme forme d'expression artistique est l'autre moyen langagier par lequel s'énonce la posture victimaire de la femme, surtout dans le contexte des conflits armés liés à la guerre asymétrique qui décime la RD Congo depuis la chute du régime de Mobutu Seseseko. Celuici avait été chassé en 1996 par la rébellion de l'AFDL (Alliance des Forces Démocratique de Libération), conduite par Laurent-Désiré Kabila. Prenons l'exemple des photos ci-après :

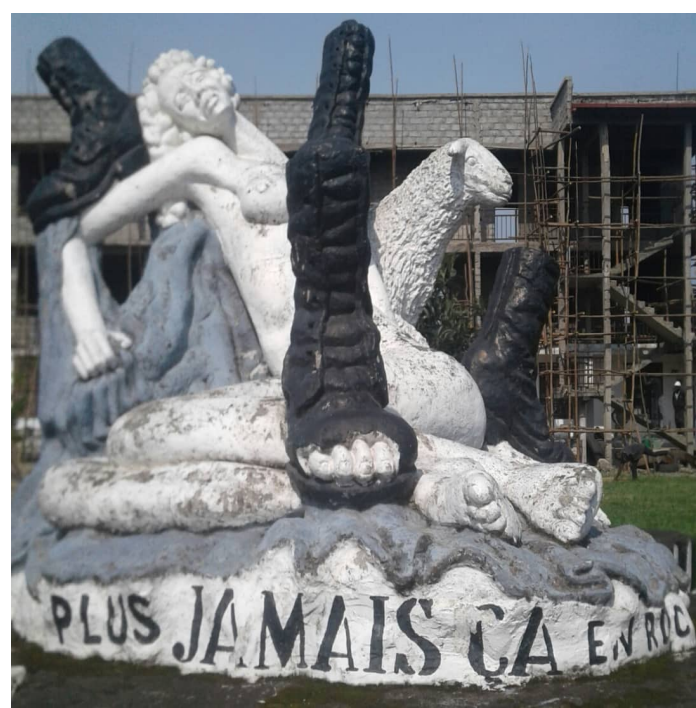

Figure 5. Image prise à Institut 


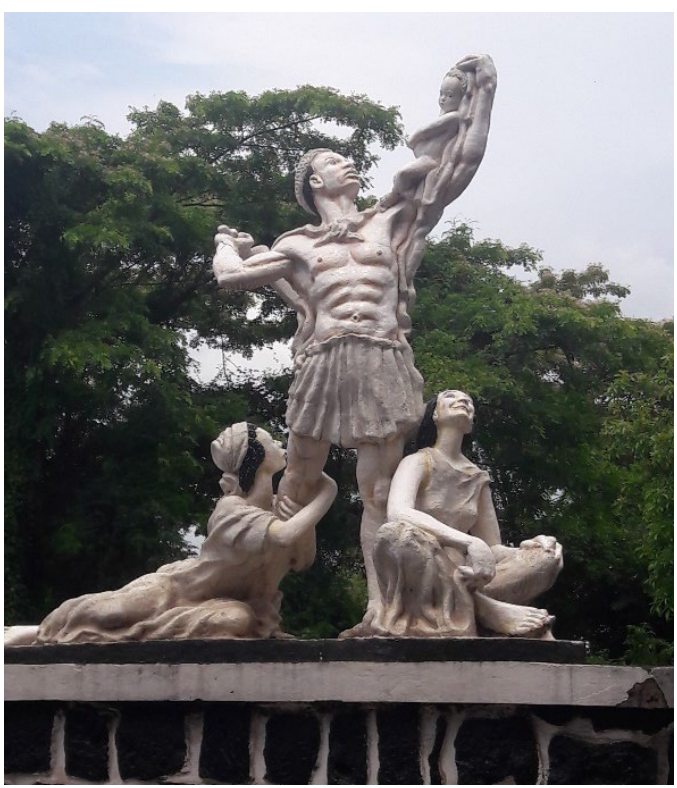

Figure 6. Image Prise à 1'ULPGL/Goma Metanoia

La figure 5 représente la mise en scène de la violence physique que l'homme fait subir à la femme. Nous y observons une femme toute nue, à la docilité d'agneau peint tout juste à côté d'elle, en train de se faire torturer par un militaire qui vient certainement à peine de la violer. Et la figure 6, quant à elle, nous peint deux femmes en train de se battre contre un homme qui tente de tuer un bébé. La teneur des discours sur le viol et les violences faites à la femme relève alors de la « rhétorique clinique » comme corollaire de l'afropessimisme contemporain, en tant que celui-ci consiste en «ce discours qui ressasse tous les maux dont souffre l'Afrique" (Mugarura Gahutu, 2014: 67). Le caractère contestataire de cette représentation sémiotique de la posture victimaire de la femme est alors étayé par le message verbal «Plus jamais ça en RDC», inscrit en bas de la Figure 5.

Le profil du pays en matière de la problématique de genre dresse le tableau sombre d'un contexte général caractérisé par un rituel de crimes indescriptibles. Du coup, la situation est au cœur des questionnements existentiels sur le destin de la femme dans une Afrique ouvertement hostile à la promotion du leadership féminin. En somme, il s'agit des productions discursives et des pratiques sociales tributaires de «l'imaginaire de la jungle » (Makomo Makita, 2014) et du sexisme culturel voire structurel qui traduit les prévarications de tout un système de gouvernance politique au 
sein de la société congolaise. En d'autres termes, la posture victimaire de la femme doit s'envisager en fonction de ses liens organiques avec les crises multiformes qui sévissent dans ce pays de l'Afrique centrale. Néanmoins, avec le mouvement international $\mathrm{du}$ "Gender mainstreaming », l'image de « victime expiatoire et résignée » de la femme d'Afrique et de la RD Congo est en train de basculer vers celle d'une héroïne dans ses représentations sémiotiques des discours sociaux.

\section{La posture de l'héroïne comme acte de renaissance des représentations sur la femme}

Dans le cadre international, l'égalité de genre qui doit se mettre en place à travers l'autonomisation de la femme et de la jeune fille ainsi qu'à travers la promotion du leadership féminin a été définie comme l'un des Objectifs de Développement Durable pour l'humanité. Au lendemain de l'adoption du plan d'action de Beijing, en 1995, l'Afrique subsaharienne a en effet connu une grande révolution constitutionnelle dans la perspective du genre. En RD Congo, la réforme du cadre normatif légal et législatif a consacré le souci d'améliorer la condition de la femme en prônant l'égalité entre les sexes, l'équité ainsi que l'autonomisation de la femme et de la jeune fille à travers la promulgation de la Constitution de Transition de 2003. Celle-ci qui prévoit la représentativité significative des femmes. Et celle du 18 février 2006 consacre la non-discrimination ainsi que la mise en œuvre de la parité homme-femme.

Dans la même dynamique, d'autres réformes législatives sont intervenues, par exemple celle du code de la famille qui a eu lieu en 2017, car jugée trop sexiste et discriminatoire à l'égard de la femme dans sa version originelle. Tout ceci sert alors de «discours constituants» (Maingueneau, 1995) pour d'autres types de discours qui positivent la femme dans la société. En RD Congo, ce renouvellement de l'imaginaire sur la femme se met en discours à travers deux types de formes sémiotiques à savoir les codes verbaux et les signes iconiques (images) empruntés à l'art pictural de l'espace urbain de Goma.

\subsection{L'expression verbale de la posture héroïne de la femme}

Dans sa mise en discours, la posture héroïne de la femme afrocongolaise s'est à tel point lexicalisée que tous les discours sociaux se sont enrichis de nouvelles expressions pour traduire cet idéal féministe. Désormais, ces discours ne figent plus les femmes dans un spectre 
socioculturel et politique négativement stéréotypé mais les représentent sous un nouveau visage de "Forces vives» de la Nation, de "Femmes leaders», de "Femmes militantes», de "Femmes résistantes», de "Femmes entrepreneuses", de "Femmes dynamiques", bref de femmes combattantes, engagées et actrices de leur propre destin. En même temps, on enregistre de plus en plus une émergence des mots et expressions figées qui sont désormais devenues des constantes des discours sociaux sur la femme. $C^{\prime}$ est entre autres des expressions telles que "Autonomisation de la femme et de la jeune fille ", "La parité homme-femme », "Leadership féminin", "Women's leadership », "Women's power », " Girls power », "Women Empowerment », etc.

Dans la logique du mouvement international francophone Genre en Action, nous avons également assisté à l'émergence exponentielle des associations féminines et collectifs des femmes dont l'action est portée par ce que nous avons convenu d'appeler le discours de militantisme associatif. De la longue liste de ces collectifs militants et associations des femmes, nous pouvons citer à titre indicatif des mouvements comme "Femmes Leaders ", "Rien Sans Les Femmes ", "Debout Fille", "Debout Congolaises ", "Observatoire de la parité », «50\% ou Rien», «Fille du Roi», "Sister to Sister», "Réseau Genre en Action», etc. Mais aussi la femme est d'ores et déjà «La Force de l'Afrique » tel que nous le lisons en swahili «NGUVU YA $A F R I C A$ » sur cette photo comme énoncé dénominatif d'une organisation féminine locale :

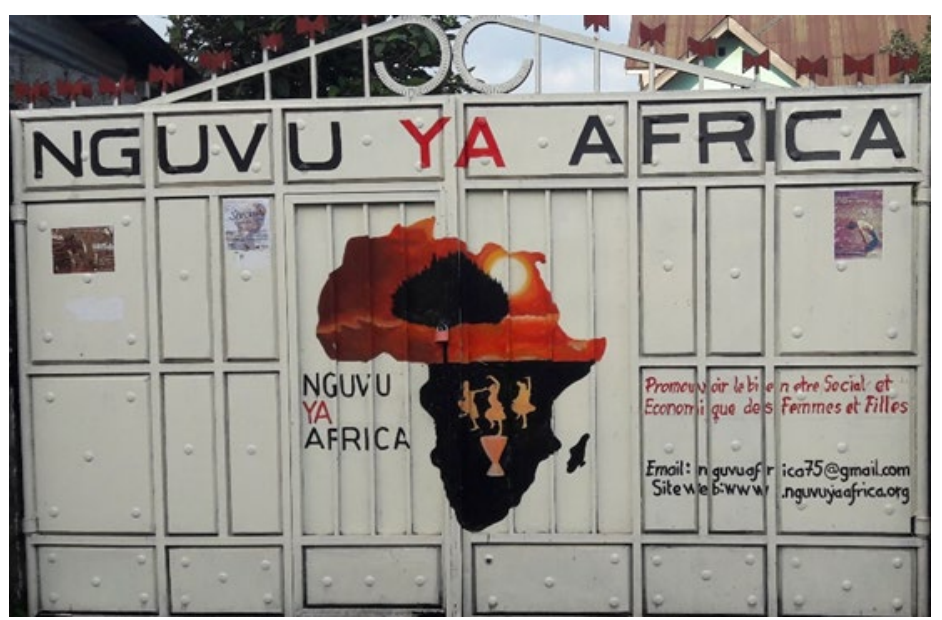

Figure 7. Photo-logo de l'ONG Nguvu ya Africa

Depuis le discours du Président de la République, prononcé le 3 mars 2019 à l'occasion de la Journée Internationale de la Femme (JIF) que le 
monde entier célèbre le 8 mars de chaque année, l'image de la femme de plus en plus médiatisée est celle d'une femme "Avenir de la Nation », tel qu'elle a été énoncée dans le mot de circonstance du Chef de l'État. Tous ces énoncés peuvent être ainsi définis comme des "mots agonistiques" (Husson A.-C., 2017) c'est-à-dire des mots de combats de cette dynamique militante autour de la femme.

\subsection{La posture héroïne de la femme au prisme de l'art pictural de l'espace urbain de Goma}

La posture héroïne de la femme congolaise est aussi à saisir dans le corpus pictural. C'est autant dire qu'une partie de l'analyse exploite certains acquis de la «rhétorique de l'image» (Barthes, 1964). Cependant, nous ne nous intéresserons pas à tous les codes plastiques de l'image. Celle-ci ne sera interprétée que par rapport à son contenu thématique (explicite ou implicite) dans son rapport avec la notion de la représentation hérö̈que de la femme. Pour illustrer cette forme de représentation sociosémiotique de la femme, nous nous sommes servis de quelques données de la production artistique picturale disponible dans la ville de Goma. En voici les deux premiers exemples retenus pour l'analyse dans le cadre de cette étude :

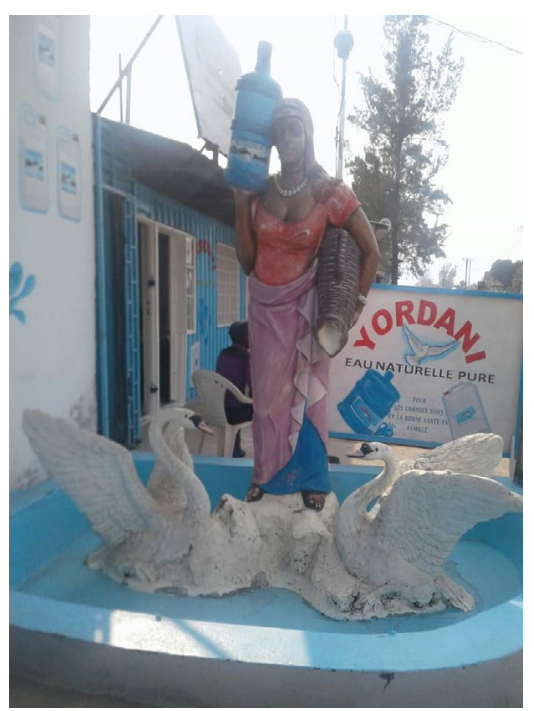

Figure 8. Photo-logo pour l'eau minéral

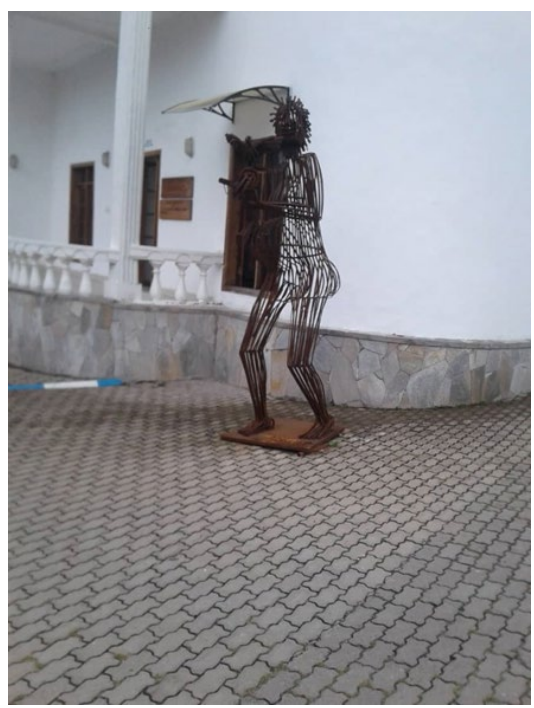

Figure 9. Image prise à l'Institut Français Yordani de Goma 
La figure 8 est un monument à fonctionnement électrique, sous forme de jet d'eau. Elle constitue une trope iconique qui se lit comme la représentation métaphorique de la femme-mère c'est-à-dire génitrice. Sur l'image, elle porte une bouteille d'eau à l'épaule. Le mécanisme a été conçu à tel point que le vase dans la main gauche est une forme de robinet d'où jaillit de l'eau potable. Dès lors, nous la saisissons comme une représentation iconique de l'expression verbale figée "L'eau c'est la vie ». L'analogie établie entre l'eau et la femme par le concepteur permet alors la glose «La femme c'est la vie ». De ce point de vue, elle est à interpréter aussi comme l'expression d'une certaine «idée reçue» (Amossy, 2010) socialement admise, selon laquelle "Les femmes sont nos mères", une traduction de l'expression figée "Banamuke ni ba mama yetu", actualisée chez les peuples swahiliphones de l'Est. D'ailleurs elle a comme variante, l'énoncé «Baza ba mères na biso" (Elles sont nos mères) que nous empruntons à la culture lingalaphone de l'Ouest.

Par contre, la figure 9 met sur la toile une femme portant une arme au canon détruit. Dans un pays en situation de guerres, entretenues par des goupes armés nationaux et étrangers, un tel message est idéologiquement très significatif. Étant donné que les acteurs de ces conflits armés sont essentiellement des hommes, l'image métaphorise par là la posture d'une femme artisanne de la non-violence et actrice de la paix, qui entend réussir là où tous les hommes ont échoué depuis des décennies, par l'approche pacifiste de son engagement. Cette interprétation est en effet plausible car au bout du canon détruit du fusil que porte la femme, se trouve une colombe comme le symbole de la paix dans la culture universelle.

Une autre production artistique picturale prise en compte consiste à représenter la femme sous l'image de personnage multifonctionnel c'est-àdire multi-solution à tous les problèmes de la famille comme noyau de base de la société. Réalisée sous l'inspiration de l'ancien Évêque de Goma, Monseigneur Théophile Kaboy, elle se présente sous la forme sémiotique ci-après : 


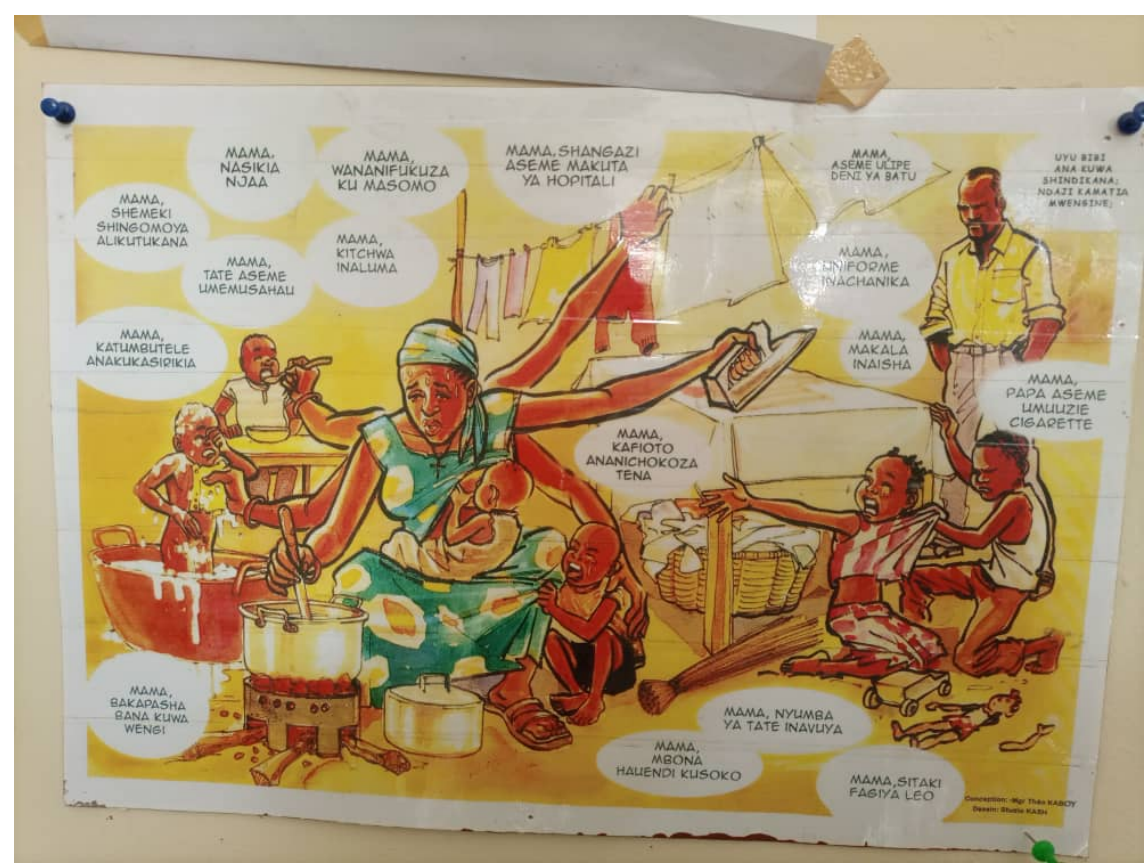

Figure 10. Image-dessin de la femme multi-solution

Le dessin ci-dessus met en scène l'image d'une femme à plusieurs bras. Dans un coin, vers l'extrême gauche se trouve un homme (son mari), debout et mains dans les poches. Nous y retrouvons également seize énoncés. Ceux-ci sont ceux qui lui sont adressés par ses enfants. Ces messages ont pour contenus, tous les problèmes aussi bien de sa famille restreinte (limitée à son foyer) que de la famille élargie. Il s'agit des messages ci-après :

(+) Mama, nasikiya njala [Maman, j'ai faim].

(+) Mama, wana nifukuza ku masomo [Maman, je viens d'être renvoyé de l'école (à cause des frais scolaires)].

(+) Mama, shangazi aseme makuta ya hopital [Maman, la tante réclame l'argent pour

son hospitalisation].

(+) Mama, aseme ulipe deni ya batu [Maman, on vous réclame de payer la dette

d'autrui].

(+) Maman, shemeki shingomoya ali kutukana [Maman, l'oncle Shingomoya vous a 
injuriée].

(+) Mama, tate aseme ume musahau [Maman, la grand-mère dit que vous l'avez

oubliée].

(+) Mama, kitchwa ina luma [Maman, j'ai des maux de tête].

(+) Mama, uniforme inachanika [Maman, mon uniforme scolaire s'est déchirée].

(+) Mama, Katumbotele ana kusirikia [Maman, Katumbotele est très fâchée contre toi]. encore].

(+) Mama, Kafyoto ana nichokoza tena [Maman, Kafyoto me provoque

(+) Mama, makala ina isha [Maman, il n'y a plus de charbon de bois (pour la cuisine)].

(+) Mama, papa aseme umu uziye cigarette [Maman, papa vous demande de lui acheter de la cigarette].

(+) Mama, ba kapasha bana kuwa wengi [Maman, les commères sont devenues très nombreuses].

(+) Mama, mbona hauwende kusoko [Maman, pourquoi ne vas-tu pas au marché ?].

(+) Mama, nyumba ya tate ina vuya [Maman, la maison de la tante suinte].

(+) Mama, sitaki fagiya leo [Maman, je ne veux pas netoyer la maison aujourd'hui].

En associant le sens implicite de cette représentation d'une femmemère à plusieurs bras à ce discours que l'enfant lui adresse, on aboutit à la posture d'une femme multi-tâches, ou bien une "machine multi-service ", un personnage extraordinaire, épique si pas extraterrestre, bref une fée aux solutions magiques pour tous les problèmes du foyer et de la société. C'est sur cette considération que repose l'hypothèse d'une "posture de l'hérö̈ne » que nous défendons, car la femme est désormais présentée comme la clé de voûte de la survie non seulement de son mari et de ses enfants, mais aussi de toute une communauté. C'est dans cette optique qu'il faut lire le discours qui présente la femme comme «La force de l'Afrique» (Figure 7). Elle est en effet la solution ultime face à l'irresponsabilité d'un père qui, se dit paradoxalement : «Uyu bibi ana kuwa shindikana. Ndajikamatiya mwengine" (Cette femme devient impossible. Je 
vais épouser une autre). C'est pourquoi la représetation de la femme-mère dans l'imaginaire de l'enfant contraste avec celle de son père ingrat et irresponsable.

Depuis la réalisation de ce dessin par le studio Kash, cette production artistique de nature picturale a donné lieu à une interprétation unique. Celle-ci l'a en effet envisagé comme l'expression symbolique d'une exploitation capitaliste de la femme par l'entreprise patriarcale. Il s'agit d'une lecture d'orientation militante féministe, induite par des déterminismes d'une tradition africaine sexiste. Pour notre part, nous estimons qu'il lui faut un regard plutôt positif. Celui-ci doit consister à le cerner comme l'expression d'une posture de femme «héroïne », pilier de l'essor socioéconomique $\mathrm{du}$ foyer, et par ricochet du développement durable de toute l'Afrique.

$\mathrm{Au}$ regard de tels supports sémiotiques, envisagés comme des «tropes iconiques » d'énociation de la posture héroïne de la femme afrocongolaise, nous pouvons bien sûr conclure que «la construction sémiotique » est un enjeu indéniable de la communication de propagandé sociétale car, selon Adam et Bonhomme (1997 : 189) :

"Les modèles sémiotiques donnent des résultats dans l'analyse des significations attachées à l'images (publicitaires), celles-ci comportant plusieurs variantes [qui permettent de considérer] le sens comme dérivé connotatif et culturel. »

Ce sont des traces d'une mémoire discursive collective, appelées à être reconfigurées, requestionnées et revalorisées dans l'optique globalisante de devoir penser le mode contemporain selon le genre. $\mathrm{Au}$ regard d'un tel processus de représention, l'appréhension de la question de l'image de la femme dans les productions discursives contemporaines exige des instances réceptrice et émitrice, la mobilisation de la trilogie de compétences « situationnelle ", " sémiologique » et "sémantique » qui a été décrite par (Maingueneau et Charaudeau, $2002: 344-346$ ).

\section{Conclusion}

Dans cette étude, il a été question de cerner les postures de la femme afro-congolaise dans ses représentations au sein des productions discursives et artistiques contemporaines en RD Congo. Le corpus qui prend en compte aussi bien l'orature parémique que les discours sociaux, littéraires, artistiques, ordinaires, etc. a permis d'établir le fait que l'imaginaire collectif jadis sexiste vis-à-vis de la femme est en train de 
connaître un certain renouvellement, en fonction des nouveaux enjeux sociaux qu'il faut inscrire dans la logique de la révolution du cadre législatif et constitutionnel congolais d'une part, ainsi que dans la dynamique des objectifs mondiaux pour Développement Durable.

Nous avons dès lors établi que la posture victimaire héritée d'une tradition fondamentalement patriarcale et donc sexiste a été essentiellement pérennisée à travers une lecture partielle c'est-à-dire subjective et décontextualisée du patrimoine parémique africain. Et c'est une lecture qui a mis en évidence une partie de ce patrimoine au discours de la négativité sur le sexe féminin. Mais une relecture objective de ce fonds culturel nous a permis de déconstruire tous les préjugés négatifs et réducteurs sur la femme, qui ont été médiatisés depuis des siècles. En fin de compte, les nouveaux discours de la renaissance des imaginaires sur la femme doivent en cela avoir comme fondement épistémologique les courants de pensée du militantisme féministe d'Afrique subsaharienne.

\section{Références bibliographiques}

1. AMOSSY, R. (1991), Les idées reçues. Sémiologie du stéréotype, Paris : Nathan.

2. BARTHES, R. (1964), Rhétorique de l'image, Paris : Seuil.

3. CHARAUDEAU, P. et MAINGUENEAU, D. (2002), Dictionnaire d'analyse du discours, Paris : Seuil.

4. HUSSON, A.-C. (2017), « Les mots agonistiques des nouveaux discours féministes : l'exemple de grossophobie et cissexisme », Argumentation et Analyse du discours [en ligne], 18/2017, disponible sur URL: http://journals.openedition.org/aad/2317, consulté le 22 avril 2021.

5. JUVET, M. (2011), Même le ciel ne pleure plus. Violences sexuelles dans la région des Grands Lacs de 1'Afrique de l'Est. Portraits et regards, Genève : Editions Slatkine.

6. MAINGUENEAU, D. (1993), Le contexte de l'œuvre littéraire, Paris : Dunod.

7. MAINGUENEA, D. (2009), Les termes clés de l'analyse du discours. Nouvelle édition revue et augmentée, Paris : Seuil.

8. MAKOMO M., J. - C. (2011), «L'état des lieux de la littérature francophone de la R.D. Congo: des pionniers aux années 2010 » in Ngorwanubusa, J. et Tognola, A. La promotion des lettres dans les pays des Grands Lacs Africains, Actes du colloque. Organisé par Sembura du 23 au 24 juin, Bujumbura : Sembura et auteurs, pp. 85-117. 
9. MAKOMO, M., J. - C. (2014), « Renouvellement de l'imaginaire littéraire des Grands Lacs Africains : regard sur quelques textes symptomatiques de la région » in Synergies Afrique des grands Lacs $n^{\circ} 3$ - 2014, pp. 93-111.

10. MUGARURA, G. A., (2014), : « Rhétorique clinique, corollaire du discours afropessimiste » IN Synergies Afrique des Grands Lacs n 3-2014, pp. 63-77.

11. TROGNON, A. et LARRUE, J. (1994), Pragmatique du discours politique, Paris : Armand-Colin. 